\title{
The anti-tumor immune response induced by a combination of MAGE-3/MAGE-n-derived peptides
}

\author{
XIU-MIN ZHANG ${ }^{1}$, YUN-FEI ZHANG ${ }^{2}$, YANG HUANG ${ }^{1}$, PING QU ${ }^{1}$, BIN MA $^{1}$, SHAO-YAN SI ${ }^{1}$, \\ ZENG-SHAN LI ${ }^{1}$, WEN-XIN LI ${ }^{1}$, XIA LI $^{1}$, WEI GE ${ }^{1}$, PEI-ZHEN HU ${ }^{1}$ and YAN-FANG SUI ${ }^{1}$ \\ ${ }^{1}$ State Key Laboratory of Cancer Biology, Department of Pathology, Xijing Hospital, Fourth Military Medical \\ University, Xi'an, Shaanxi Province 710032; ${ }^{2}$ Center of Orthopaedic Surgery, Orthopaedics Oncology Institute of \\ Chinese PLA, Tangdu Hospital, Fourth Military Medical University, Xi'an, Shaanxi Province 710038, P.R. China
}

Received January 21, 2008; Accepted April 22, 2008

\begin{abstract}
Tumor antigen-derived peptides have been widely used to elicit tumor-specific cytotoxic T lymphocytes (CTLs). MAGE gene products are of particular interest owing to their wide expression in many tumors and their potential to induce tumor-specific CTL responses. Antigen-specific CTLs induced by MAGE gene-derived peptides have proven to be highly efficacious in the prevention and treatment of various types of tumors. MAGE-3 has been used as a target for tumor immunotherapy. MAGE- $n$ is a new member of the MAGE gene family and has been shown to be closely associated with hepatocellular carcinoma (HCC). However, the majority of previous investigations focused on the single MAGE antigen-derived peptides as a cancer vaccine which has many limitations. The tumor antigen expression is known to be heterogeneous and tumor cells can express multiple tumor antigens. Thus, vaccines incorporating single antigen-derived epitopes may be inadequate in generating a complete immune response against the tumor. Instead, a polyvalent vaccine incorporating epitopes derived from several tumor antigens may be more effective. Our study combined the MAGE-3 and MAGE-n-derived peptides as a cancer vaccine. The results showed that the combination of MAGE-3 and MAGE-n epitopes induced more effective anti-tumor immune responses than either of the peptides alone. In addition, the peptidespecific activity was observed to be in an MHC-restricted manner. Our study indicated that the combination of several tumor antigen-derived peptides may present a better peptidebased cancer immunotherapy.
\end{abstract}

Correspondence to: Dr Yan-Fang Sui, State Key Laboratory of Cancer Biology, Department of Pathology, Xijing Hospital, Fourth Military Medical University, Xi'an, Shaanxi Province 710032, P.R. China

E-mail: suiyanf@163.com

Key words: tumor antigen, MAGE-n, MAGE-3, cytotoxic T lymphocyte epitope, enzyme-linked immunosorbent spot

\section{Introduction}

Hepatocellular carcinoma (HCC) is one of the most common malignant tumors with a high rate of recurrence and metastasis. However, conventional treatments such as surgery, chemotherapy and radiotherapy are palliative and cannot eradicate the tumor cells, so new therapies are urgently required. Cancer immunotherapy is a new promising treatment. The recent identification and molecular characterization of tumor-associated antigens recognized by tumor-reactive $\mathrm{CD}^{+}$lymphocytes has facilitated the development of immunotherapy protocols against cancer types.

The MAGE gene family was the first reported example of these tumor-associated antigen genes, which are expressed in a variety of malignant neoplasms, and in the testis as the only normal tissue (1). Antigen-specific CTL induced by MAGEderived peptides has proven to be highly efficacious in the prevention and treatment of various types of tumors (2-4). MAGE-3 is expressed in a significant proportion of primary and metastatic tumors of various histological types, such as in $74 \%$ of metastatic melanoma and in $50 \%$ of carcinomas of the esophagus, head and neck, bladder and lung (5). The previously reported clinical effectiveness of MAGE-3.A1derived peptides are encouraging. The MAGE- $n$ is a new member of the MAGE gene family, which was reported for the first time by our lab (Genebank, locus No. AF443295) (6). It was highly homologous to the MAGE-A subfamily genes, especially to MAGE-3 (93\%). It has been proven that the MAGE-n-derived peptide QLVFGIEVV was a new HLAA2.1-restricted CTL epitope capable of inducing MAGE-nspecific CTLs in vitro (7). The identification of this CTL epitope opens up the possibility of clinical applications of this peptide-based cancer vaccine for patients with MAGE- ${ }^{+}$.

In most studies performed thus far, single tumor antigenderived peptides have been administered, sometimes in various formulations (8-14). Alternatively, several peptides derived from a single antigen have been administered simultaneously (15). However, tumor antigen expression is known to be heterogeneous, even in the same tumor (16). Tumor cells can express multiple tumor antigens and the expression of each of these can vary within the tumor. Therefore, a vaccine containing peptides derived from several of these antigens 
may be more effective than a vaccine directed against a single antigen. The polyvalent vaccine can effectively enhance its immunogenicity and induce stronger CTL activity (17-19). In the present study, we investigated the combination of MAGE-3 and MAGE-n-derived peptide-based cancer vaccine. In view of the fact that HLA-A2 is one of the most frequently expressed molecules in China (20), we investigated the combination of the best epitope of MAGE-3/HLA-A2+ and MAGE-n/HLA-A2 ${ }^{+}$with epitope reconstruction and immunological methods. In the present study, MAGE-3 and MAGE-nspecific CTLs were induced from the peripheral blood mononuclear cells (PBMC) of healthy donors by multiple stimulations with HLA-A2-restricted MAGE-3-derived peptides, MAGE-n-derived-peptides or a combination of MAGE-3/MAGE-n-derived peptide-pulsed T2 cells. Then, the immunological activities of the HLA-A2-restricted CTL epitopes of MAGE-3, MAGE-n and the combined epitopes of MAGE-3 and MAGE-n were analyzed by the epitope reconstruction method, enzyme-linked immunosorbent spot assay (ELISPOT) and specific cytotoxicity assay. The results showed that the combination of MAGE-3 and MAGE-n epitope elicited a stronger specific anti-tumor immunity than either peptide alone.

\section{Materials and methods}

Cell lines. T2 cells, which bear the HLA-A*0201 gene, but express a very low level of cell surface HLA-A2.1 molecules and are unable to present endogenous antigens due to a deletion of most of the MHC class II region including TAP (transporter-associated with antigen processing) and proteosome genes, were kindly provided by Dr Sun Zongtang (Chinese Academy of Medical Sciences). Hepatocellular carcinoma cell lines HHCC, gastric carcinoma cell lines KATO-III and breast carcinoma cell lines MCF-7 were all HLA-A2 ${ }^{+}$and cultured in our lab (20-23). All these cell lines were maintained in RPMI-1640 medium (Invitrogen, USA) supplemented with $12 \%$ fetal bovine serum (FBS, Hyclone, USA) and antibiotics.

Synthetic peptides. The HLA-A2-restricted CTL epitopes MAGE-3 $271-279$ (FLWGPRALV), MAGE-n ${ }_{159-167}$ (QLVFGIEVV) and influenza virus matrix protein (Flu-MP) (GILGFVFTL) peptides were synthesized by standard solid-phase chemistry and characterized by mass spectrometry. A high purity peptide synthesis with a purity of $>90 \%$ was indicated by analytical HPLC. The flu-MP peptide was selected as a positive control. Lyophilized peptides were dissolved in DMSO and stored at $-80^{\circ} \mathrm{C}$ after being diluted in PBS.

Expression of the MAGE-3 and MAGE-n gene in tumor cell lines were detected by RT-PCR. The expression of MAGE-3 and MAGE-n mRNA were detected with RT-PCR. Primers for the MAGE-3 gene were: upstream 5'-ATGCCTCTTGAGCA GAGGAG-3' (M1) and downstream 5'-GCTCACTCTTCC CCCTCTCTC-3' (M2). Primers for the MAGE-n gene were: upstream 5'-AGCAACCAGGAAGAGGAGGGGCCAAG CAC-3' (Pn1) and downstream 5'-TAAATCGTAGGAGA GGCCCAGGCAGGTGACAAG-3' (Pn2). The $\beta$-actin primers have also been synthesized as a positive control: upstream
5'-AGCAAGAGAGGCATCCTCAC-3' (ß-actin 1) and downstream 5'-AGCTCGTAGCTCTTCTCCAG-3' (ß-actin 2). Total RNA was extracted from $\sim 1 \times 10^{7}$ tumor cells in TRIzol reagent (Life Technologies, USA), following the recommended protocols. Briefly, total RNA was incubated at $50^{\circ} \mathrm{C}$ for $30 \mathrm{~min}$, to prepare the first chain of cDNA for the detection of MAGE-3 and MAGE-n. The PCR process of MAGE-3 was: predenaturation at $94^{\circ} \mathrm{C}$ for $5 \mathrm{~min}$, denaturation at $94^{\circ} \mathrm{C}$ for $30 \mathrm{sec}$, annealing at $55^{\circ} \mathrm{C}$ for $30 \mathrm{sec}$ and extension at $72^{\circ} \mathrm{C}$ for $60 \mathrm{sec}, 30$ circles and a final extension at $72^{\circ} \mathrm{C}$ for $10 \mathrm{~min}$. The PCR process of MAGE-n was: predenaturation at $94^{\circ} \mathrm{C}$ for $5 \mathrm{~min}$, denaturation at $94^{\circ} \mathrm{C}$ for $30 \mathrm{sec}$, annealing at $55^{\circ} \mathrm{C}$ for $40 \mathrm{sec}$ and extension at $72^{\circ} \mathrm{C}$ for $40 \mathrm{sec}, 30$ circles and a final extension at $72^{\circ} \mathrm{C}$ for $10 \mathrm{~min}$. The predicted size of the amplified segment of the MAGE-3 gene was $950 \mathrm{bp}$, and that of the MAGE-n gene was $303 \mathrm{bp}$. B-actin was amplified as the internal control and the amplified segment of $\beta$-actin was $530 \mathrm{bp}$. The final products were examined on a $1.5 \%$ agarose gel.

Establishment and expression of the HHCC cell line with MAGE-3 gene. Hepatocellular carcinoma cell lines, HHCC, which had no homologous form of MAGE-3 were transfected with the plasmid pcDNA-MAGE-3 using Lipofectamine 2000 (Invitrogen, USA) and then selected in the presence of $800 \mu \mathrm{g} / \mathrm{ml} \mathrm{G} 418$ (Sigma Chem. Co.). The G418-resistant clones were subcloned and then screened for MAGE-3 expression by RT-PCR using the MAGE-3 primers. The positive $\mathrm{HHCC}+\mathrm{MAGE}-3$ cells were maintained at $37^{\circ} \mathrm{C}$ in $5 \% \mathrm{CO}_{2}$ in RPMI-1640 containing $15 \%$ fetal bovine serum and $400 \mu \mathrm{g} / \mathrm{ml} \mathrm{G} 418$.

The TRIzol method was used to isolate RNA in HHCC+MAGE-3 cells. The expression of MAGE-3 and MAGE-n mRNA was detected with RT-PCR with M1M2 and Pn1Pn2 as primers. Part of the products underwent $10 \mathrm{~g} / \mathrm{l}$ agarose gel electrophoresis.

Induction of CTL. PBMCs were obtained from a healthy HLA-A2 ${ }^{+}$donor with informed consent and separated using Ficoll-Hypaque gradient density centrifugation (Sigma, USA). The HLA-A typing of PBMCs was performed by using FACScan with anti-HLA-A2 MAb (US Biological, USA).

The PBMCs were respectively stimulated in vitro with MAGE-3, MAGE-n, MAGE-3+MAGE-n or flu peptidepulsed T2 cells according to the previous protocol (24). Briefly, T2 cells $\left(1 \times 10^{5} / \mathrm{ml}\right)$ were washed three times in serum-free medium and incubated with MAGE-3, MAGE-n, MAGE-3+MAGE-n or flu epitope peptides, respectively, at a concentration of $50 \mu \mathrm{g} / \mathrm{ml}$ for $2 \mathrm{~h}$ at room temperature. ß2-microglobulin ( $\beta_{2}-\mathrm{m}$, Sigma) was added at a concentration of $3 \mu \mathrm{g} / \mathrm{ml}$. The peptide-pulsed T2 cells were then irradiated with $7500 \mathrm{cGy}$, washed once and added to freshly isolated PBMCs in RPMI-1640 supplemented with $15 \%$ heatinactivated fetal bovine serum. IL-2 (100 U/ml, R\&D, UK) was added the following day. After a 7-day co-culture, the second stimulation was performed. The cultured cells were maintained by weekly stimulation with peptide-pulsed T2 cells. After a total of four-time stimulations in vitro, the responder cells were tested for cytotoxic activity against the target cells. 
Phenotype analysis. The phenotypes of responder cells were assessed using flow cytometry. The cells were stained with FITC-conjugated mouse anti-human CD3 mAb, phycoerythrin (PE)-conjugated mouse anti-human CD4 mAb and Per CPconjugated mouse anti-human CD8 mAb (Becton-Dickinson, USA). Isotype-matched mouse antibodies served as a negative control. Stained cells were detected using FACScan (BectonDickinson).

IFN- $\gamma$ enzyme-linked immunosorbent spot (ELISPOT) assays. To determine the frequency of $\mathrm{T}$ cells capable of responding to a specific stimulus by the secretion of IFN- $\gamma$, an ELISPOT assay has been established by the human IFN- $\gamma$ ELISPOT system (Diaclone, France). The ELISPOT assay was performed according to the manual in wells of 96-well flat-bottom microtiter polystyrene enzyme-linked immunosorbent assay plates coated with $5 \mu \mathrm{g}$ of capture anti-IFN- $\gamma \mathrm{Ab} / \mathrm{ml}$ in $100 \mu \mathrm{l}$ of the diluents (PBS, pH 7.2) per well. The plates were incubated overnight at $4^{\circ} \mathrm{C}$, washed extensively with PBS and blocked with $2 \%$ skimmed dry milk in PBS for $2 \mathrm{~h}$ at room temperature. Purified responder cells resuspended in RPMI1640 medium without PBS were added at $1 \times 10^{5}$ cells/well in triplicate. The stimulatory peptides (MAGE-3, MAGE-n, MAGE-3 and MAGE-n or flu peptide) pulsed onto irradiated presenting cells (T2) were then added to each well $\left(2 \times 10^{4}\right.$ cells/well) and the plates were incubated for $18-20 \mathrm{~h}$ at $37^{\circ} \mathrm{C}$. The plates were then vigorously washed with $0.1 \%$ Tween-20 PBS and the biotinylated detection anti-IFN- $\gamma \mathrm{Ab}$ was added at $5 \mu \mathrm{g} / \mathrm{ml}$. The plates were again incubated for $2 \mathrm{~h}$ at $37^{\circ} \mathrm{C}$. Following washing, streptavidin-conjugated alkaline phosphatase was added at $37^{\circ} \mathrm{C}$ for $1 \mathrm{~h}$. Individual cytokineproducing cells were detected as dark spots after a 20-min reaction with ready-to-use BCIP/NBT. The number of dark spots/well was determined microscopically. The frequency of positive (IFN- $\gamma$-producing) cells was calculated after the number of spots in the control wells had been subtracted from that in the experimental wells. The control wells contained non-stimulated effector cells (spontaneous IFN- $\gamma$-production). The background frequency was $4 / 1 \times 10^{5}$ PBMC.

Cytotoxicity assays. The colorimetric CytoTox 96 assay (Promega, USA) was used to quantify the release of a cytosolic enzyme, lactate dehydrogenase, upon target cell lysis. Tumor cell lines HHCC, KATO-III, HHCC-MAGE-3 and MCF-7 cells were selected as target cells. K562 cells were selected as the negative control. Other target cells such as T2+MAGE-3/MAGE-n/MAGE-3+MAGE-n (the peptidepulsed $\mathrm{T} 2$ cells), were prepared by incubating T2 cells with MAGE-3, MAGE-n, MAGE-3+MAGE-n or flu epitope peptides $(50 \mu \mathrm{g} / \mathrm{ml})$ for $2 \mathrm{~h}$ at room temperature. A constant number of target cells $\left(1 \times 10^{4} / 100 \mu \mathrm{l}\right)$ was added to the serial dilution of various effectors for a 4-h incubation at $37^{\circ} \mathrm{C}$. After centrifugation, 50- $\mu 1$ aliquots of cell-free supernatant were assayed for LDH content. To correct for spontaneous LDH release from the effector cells, LDH levels were measured for each individual effector cell concentration used in the experimental setup (effector spontaneous). The target cell spontaneous LDH release (target spontaneous) was also measured. The maximum target cell LDH release (target maximum) was measured after cell lysis with $0.4 \%$ Triton

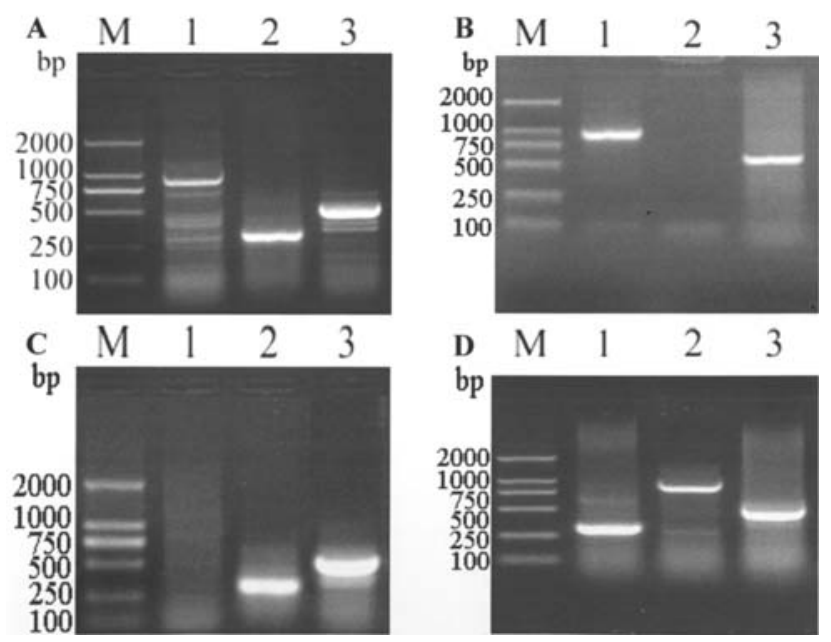

Figure 1. The expression of MAGE-3 and MAGE-n gene in tumor cell lines as shown by RT-PCR results. (A) MAGE-3 and MAGE-n in KATO-III cells. (B) MAGE-3 and MAGE-n in MCF-7 cells. (C) MAGE-3 and MAGE-n in HHCC cells. M, PCR marker (DL2000); lane 1, MAGE-3; lane 2, MAGE-n and lane 3, B-actin. (D) The expression of MAGE-3 and MAGE-n gene in HHCC-MAGE-3 cells as shown by RT-PCR results. M, PCR marker (DL2000); lane 1, HHCC cells transfected with MAGE-n; lane 2, HHCC cells transfected with MAGE-3 and lane 3, RT-PCR product of $\beta$-actin.

$\mathrm{X}-100$ and considered as $100 \% \mathrm{LDH}$ release. For experiments performed in serum-free AIM-V medium, effector cells were washed twice in AIM-V medium before adding to the target cells. The measured values were assayed in triplicate and corrected for the culture medium LDH background. The percentage of specific LDH release was determined as the formula:

Experimental-Effector-Spontaneous-Target-Spontaneous

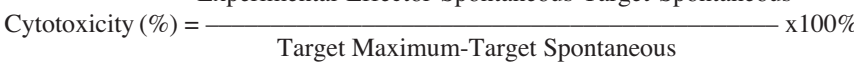

Inhibition of cytotoxicity with monoclonal antibodies. Appropriate target cells were incubated with monoclonal antibodies at a final concentration of $1 / 20$ for $1 \mathrm{~h}$ at $4^{\circ} \mathrm{C}$ prior to the assay of cytotoxicity. To determine inhibition, an antiHLA-A2 antibody (Immunotech, France) or a control mAb (mouse immunoglobulin G2, IgG2, Caltag) was added to the peptide-loaded target cells. After incubation, the target cells were mixed with effector cells for the LDH release assay. MCF-7, HHCC, KATO-III and HHCC-MAGE-3 were used as target cells.

Statistical analysis. The data in this study were analysed using SPSS software. Differences with $\mathrm{p}<0.05$ were considered significant.

\section{Results}

The MAGE-3 and MAGE- $n$ gene expression in tumor cell lines. The HHCC+MAGE-3 cell line was established by liposome transfection. MAGE-3 and the MAGE-n gene were expressed in KATO-III and HHCC+MAGE-3 cells (Fig. 1A and D). In MCF-7 cells, only the MAGE-3 gene was expressed and in the HHCC cells, only the MAGE-n gene was expressed (Fig. 1B and C). 

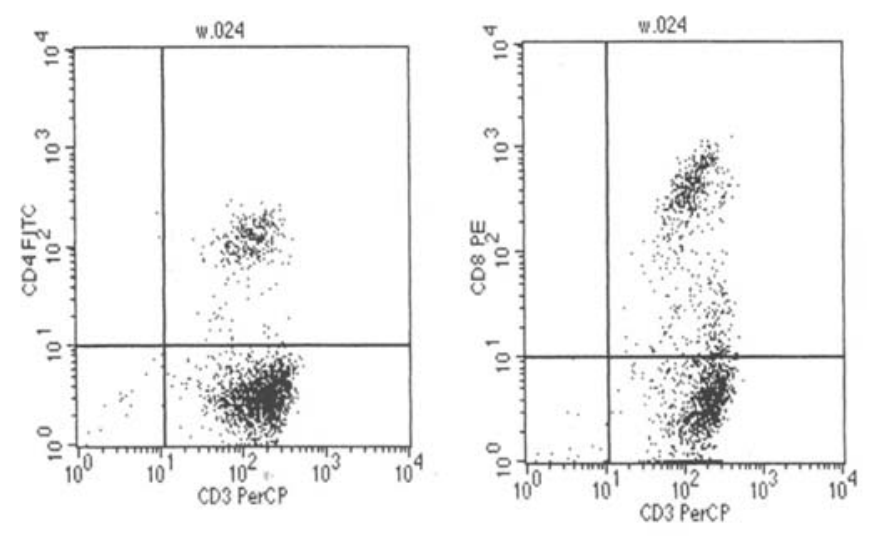

Figure 2. Phenotypic analysis of induced T cells (on the 24th day of culture). The percentage of $\mathrm{CD}^{+}$cells gradually decreased while the $\mathrm{CD} 8^{+}$cells increased. On the 24 th day of culture, $>97 \%$ of these cells were $\mathrm{CD}^{+}$and $\sim 68 \%$ were $\mathrm{CD}^{+}$

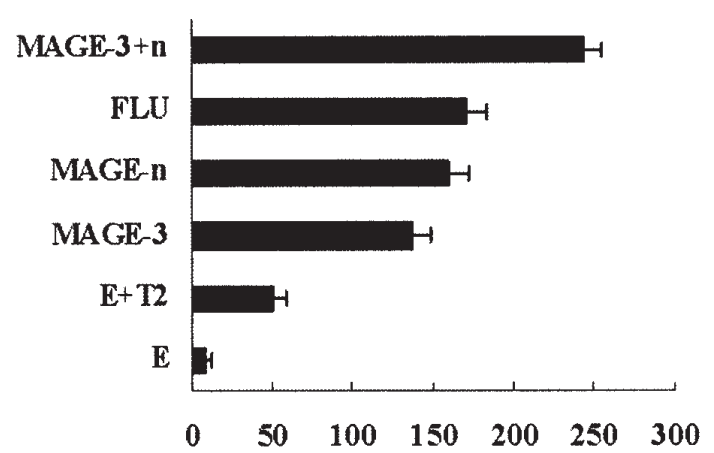

Figure 3. ELISPOT detection of PBMC stimulated by peptide-pulsed T2 cells. PBMC were cultured overnight with T2 pulsed MAGE-3, MAGE-n, MAGE3+MAGE-n/HLA-A2 peptides or the Flu/HLA-A2 peptide in a standard IFN-ELISPOT assay. E, Effector; E+T2, effector induced by T2 cells; MAGE-3/MAGE-n/MAGE-3+n, effector induced by T2 cell-loaded MAGE-3/MAGE-n/MAGE-3+MAGE-n peptides and FLU, effector induced by $\mathrm{T} 2$ cell-loaded Flu peptide.

Growth kinetics and the characterization of the induced T cells. Following the first stimulation using the T2 cells pulsed MAGE-3+MAGE-n peptide, the PBMCs were expanded continuously. As a result, a 30-40-fold increase of the cell number was achieved on the 24th day of culture. A flowcytometric analysis of the cells was performed before and after the culture. Before culture, the frequency of $\mathrm{CD}^{+}, \mathrm{CD}^{+}$ and $\mathrm{CD}^{+}$cells were 35,20 and $17 \%$, respectively. However, the number of $\mathrm{CD}^{+}$cells gradually increased with the culture. The percentage of $\mathrm{CD}^{+}$cells gradually decreased while the $\mathrm{CD} 8^{+}$cells increased. On the 24 th day of culture, $>97 \%$ of these cells were $\mathrm{CD}^{+}$and $\sim 68 \%$ were $\mathrm{CD}^{+}$(Fig. 2).

ELISPOT. The results of IFN- $\gamma$ ELISPOT demonstrated that the association of the MAGE-3 and MAGE-n epitope induced more specific $\mathrm{T}$ cells than other groups $(\mathrm{p}<0.05)$. The tumor cell lines used in this study were HLA-A2 ${ }^{+}$cells. The average IFN- $\gamma$ spot number of responding lymphocytes, induced by T2 pulsed with a single epitope HLA-A2 peptide, was much higher than that induced by T2 cells without a peptide $(\mathrm{P}<0.05)$. (Fig. 3, Table I).
Table I. The spot number of PBMC stimulated by peptidepulsed T2 cells.

Groups IFN- $\gamma$ spots $/ 1 \times 10^{5}$ splenocyte

\begin{tabular}{lc}
\hline E & $8.5 \pm 3.5$ \\
E+T2 & $51.0 \pm 8.5$ \\
MAGE-3 & $137.0 \pm 11.3$ \\
MAGE-n & $160 \pm 12.7$ \\
FLU & $170.5 \pm 13.4$ \\
MAGE-3+n & $243.5 \pm 12.0^{\mathrm{a}}$
\end{tabular}

${ }^{a}$ The average IFN- $\gamma$ spot number of responding lymphocytes, induced by $\mathrm{T} 2$ pulsed with MAGE-3 and MAGE-n epitope HLA-A2 peptide, was much higher than other groups $(\mathrm{P}<0.05)$.

Cytotoxic activity of the induced CTL cells. HHCC, KATO-III and MCF-7 were all HLA-A2 ${ }^{+}$as previously reported (20-22). Our results showed the MAGE-n gene was expressed in KATO-III and HHCC+MAGE-3 cells. In MCF-7 cells, only the MAGE-3 gene was expressed, and in HHCC cells, only the MAGE-n gene was expressed. To analyze the specific cytotoxicity of CTLs induced by T2 pulsed with MAGE-3, MAGE-n or MAGE-3+MAGE-n HLA-A2-restricted peptides, the cytotoxicity assay was performed using these cell lines. The lysis of CTLs against T2+MAGE-3, MCF-7, KATO-III and HHCC+MAGE-3 (MAGE- $3^{+}$) induced by T2 pulsed with the MAGE-3 peptide were significantly higher than that against HHCC (MAGE-3-) (Fig. 4A). CTL lysis against T2+MAGE-n, HHCC, KATO-III and HHCC+MAGE-3 $\left(\right.$ MAGE- $\left.{ }^{+}\right)$induced by $\mathrm{T} 2$ pulsed with a MAGE-n peptide were significantly higher than that against MCF-7 (MAGE-n') (Fig. 4B). CTLs induced by MAGE-3 and MAGE-n peptides significantly lysed HHCC, HHCC+MAGE-3, KATO-III and MCF-7 cell lines (Fig. 4C).

Inhibition of the recognition of the induced effectors by monoclonal antibodies. To determine whether the induced CTL cells recognized the MAGE-3, MAGE-n and MAGE3+MAGE-n expressing target in a HLA class-I-restricted manner, monoclonal antibodies generated against HLA-A2 molecules were utilized to block the cytotoxicity of effectors. The cytotoxic activity of the effector cells (MAGE-3, MAGE-n and the MAGE-3+MAGE-n peptide-induced CTLs) against the MCF-7, HHCC, KATO-III and HHCC+MAGE-3 were significantly eliminated by the anti-(HLA-A2) antibody, (Fig. 5A-C). These data suggested that the induced CTLs lyse the targets expressing MAGE-3, MAGE-n and MAGE3+MAGE-n in a HLA-A2-restricted manner.

\section{Discussion}

Recent studies show that tumor antigens, especially the tumorspecific antigen (TSA), which can induce tumor-specific CTL and damage tumor cells, are a major component of tumor vaccines. Among the tumor rejection antigens identified thus far, MAGE gene products are of particular interest owing to their wide expression in many tumors and their potential to 

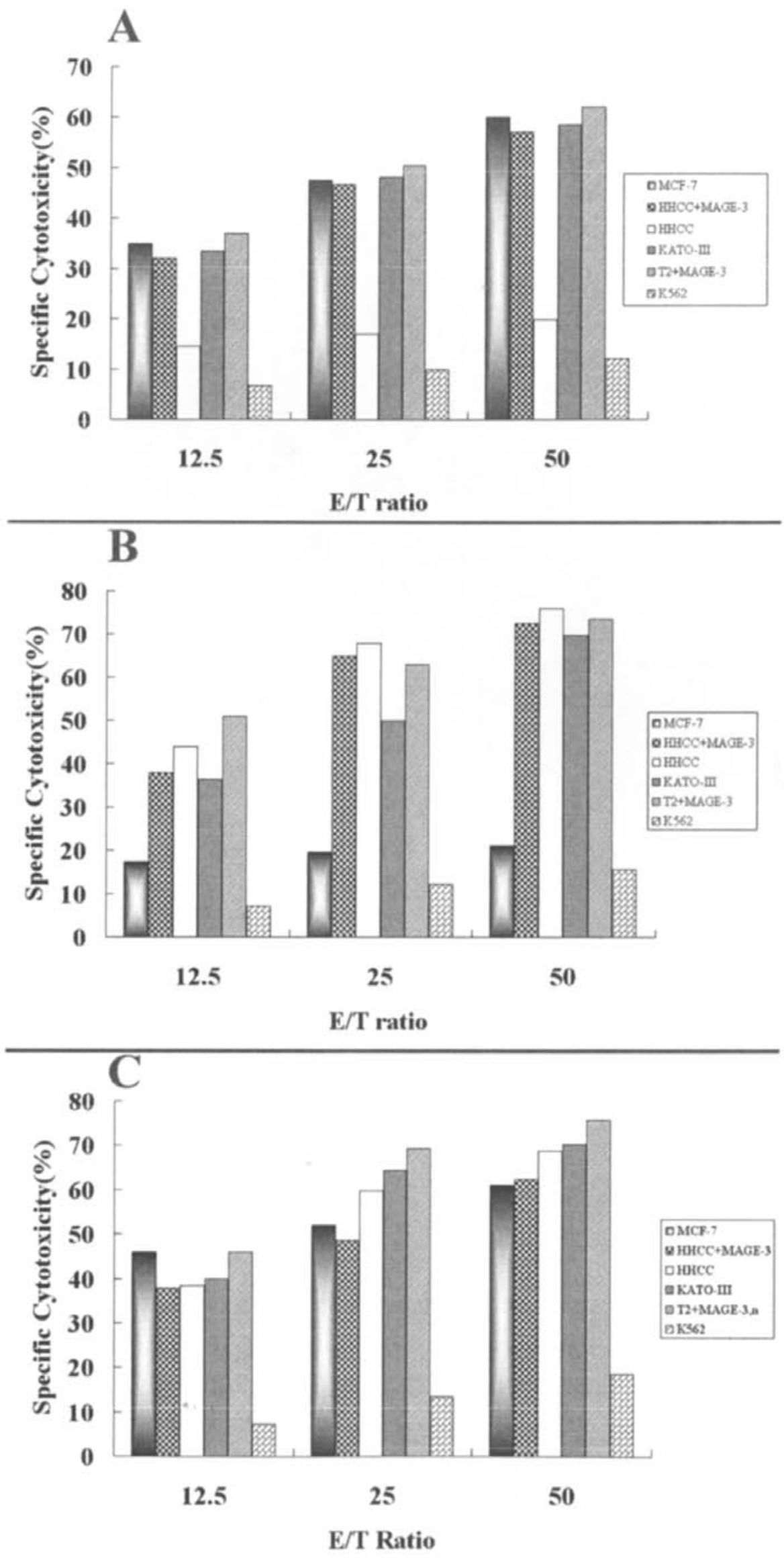

Figure 4. Lysis of various cell lines by the CTL cells induced from the PBMC using MAGE-3, MAGE-n and MAGE-3+MAGE-n/HLA-A2 peptides. The CTLs were obtained by stimulating the PBMC with MAGE-3 peptide-pulsed T2 cells for 24 days. (A-C) Cytotoxicity of MAGE-3, MAGE-n or MAGE-3+MAGE-n peptide-induced CTLs. The cytotoxic activities of the CTLs against KATO-III, HHCC-MAGE-3 (HLA-A2 ${ }^{+}$, MAGE- $3^{+}$and MAGE- ${ }^{+}$), MCF-7 (HLA-A2 ${ }^{+}$and MAGE- $3^{+}$), HHCC (HLA-A2 ${ }^{+}$and MAGE- ${ }^{+}$), T2+MAGE-3 (the peptide-pulsed T2 cells, HLA-A2 ${ }^{+}$and MAGE- $3^{+}$), T2+MAGE-n (the peptide-pulsed T2 cells, HLA-A2 ${ }^{+}$and MAGE- ${ }^{+}$), T2+MAGE-3, n (the peptide-pulsed T2 cells, HLA-A2 $2^{+}$, MAGE- $3^{+}$and MAGE- ${ }^{+}$) and K562 cells were assessed at various E:T ratios. 

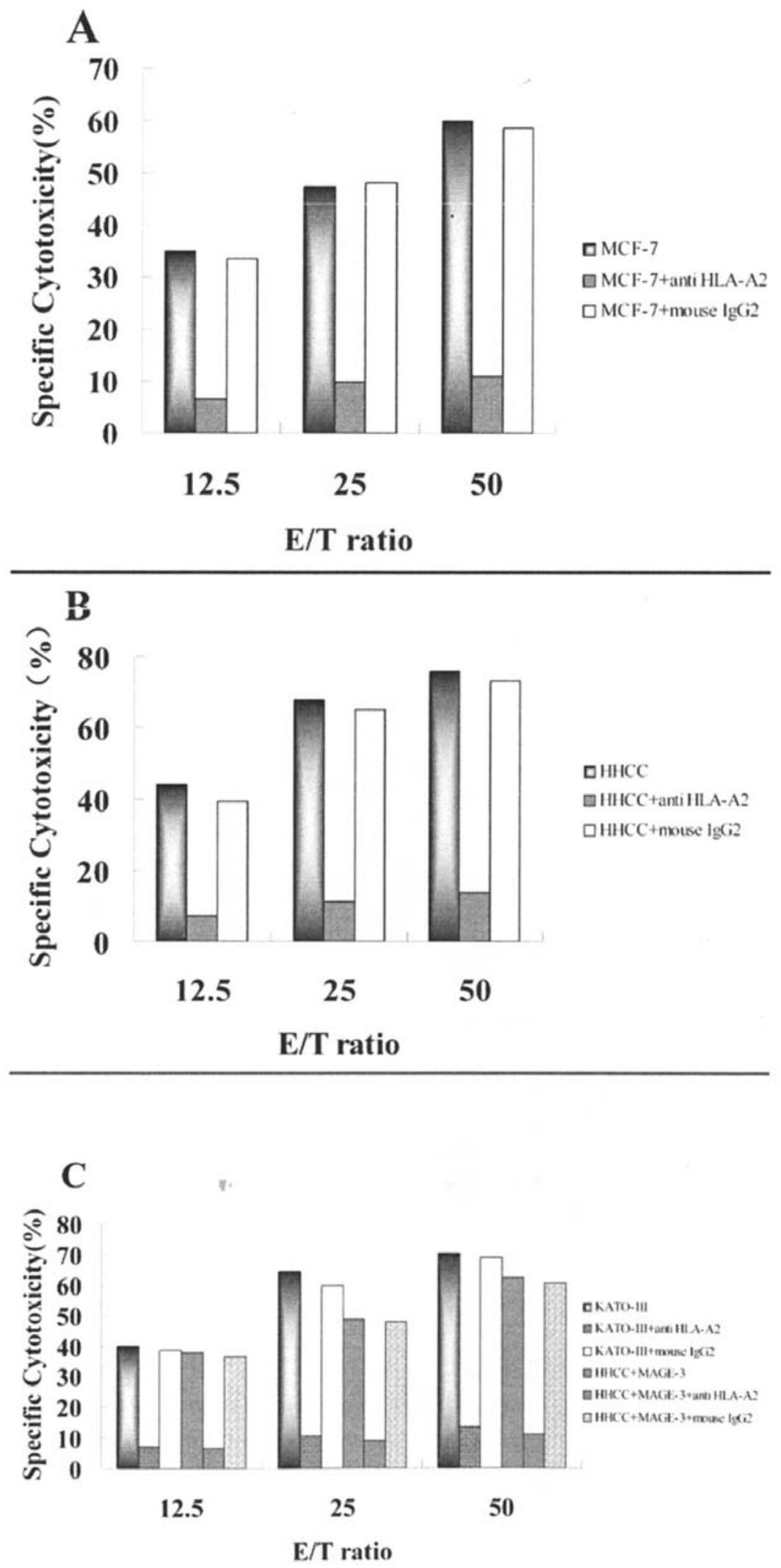

Figure 5. (A-C) Inhibition of the cytotoxicity activity of the effector cells (MAGE-3, MAGE-n or MAGE-3+MAGE-n peptide-induced CTLs) by anti-HLA-A2 monoclonal antibodies. The target cells pretreated with anti-HLA-A2 antibody or a control mAb, in the absence of antibodies for $4 \mathrm{~h}$. The cytotoxic activity of the effectors was then assessed at various E:T ratios.

induce tumor-specific CTL responses. The MAGE gene family-related genes, such as MAGE-1, $-2,-3,-4$ and -6 , are expressed in many tumors and code for antigens that can be presented in association with some HLA class I alleles (25).
In most of the previous reports, single tumor antigenderived peptides were administered. Though the effective antitumor immune responses have been induced, the use of single tumor antigen-derived peptides have many limitations. 
As we know, tumor cells can express multiple tumor antigens and the expression of each of these can vary within the tumor. Thus, vaccines incorporating single antigen-derived epitopes may be inadequate in generating a complete immune response against the tumor $(2,26)$. Instead, a polyvalent vaccine incorporating epitopes derived from several of these antigens may be more effective than a vaccine directed against a single antigen. In addition, the elicitation of immune responses directed against several antigens can minimize the risk of selecting antigen loss tumor variants (27).

In this study, single or combined HLA-A2-restricted CTL epitopes of MAGE-n and MAGE-3 were identified by epitope reconstruction and immunological assay. The ability of epitopespecific CTLs to suppress the growth of human tumors ex vivo was studied. Initially, we got HLA-A2+ PBMCs from healthy donors by Ficoll discontinuous density gradient isolation. The peptide-specific CTLs were then successfully induced from the PBMCs by multiple stimulations with MAGE peptide-pulsed T2 cells. The ELISPOT assay showed that the average IFN- $\gamma$ spot numbers of responding lymphocytes induced by $\mathrm{T} 2$ pulsed with MAGE- 3 and the MAGE-n combined peptide were much higher than that of responding lymphocytes in other groups. The average IFN- $\gamma$ spot numbers of responding lymphocytes induced by $\mathrm{T} 2$ pulsed with a MAGE-3 or MAGE-n single peptide were higher than that of responding lymphocytes induced by $\mathrm{T} 2$ cells without a peptide.

LDH assay results showed that CTLs stimulated with combined peptides of MAGE- 3 and MAGE-n exhibited a specific lysis of MCF-7, HHCC, HHCC+MAGE-3, KATO-III and T2+MAGE-3 and MAGE-n cell lines. When the ratio of effector to target cells was 50:1, the killing rate of CTLs was $61,68.8,62.3,70.2$ and $75.8 \%$ respectively. However, CTLs stimulated with a single peptide of MAGE-3 or MAGE-n exhibited a specific lysis of cell lines only with a corresponding peptide, indicating that a combination of epitopes derived from several antigens may enhance the immunity and antitumor effect compared with the vaccine directed against a single antigen.

Two criteria are important to consider when selecting peptides for a vaccine to be tested in the majority of tumors. The first is the frequency of expression of the parent protein from which the epitope is derived, and the second is the frequency of expression of the HLA alleles to which the epitopes are restricted. MAGE-3 and MAGE-n are expressed in a large percentage of HCC. The epitopes described in this study are restricted by HLA-A2 molecules, which is one of the most frequently expressed molecules in China.

MAGE epitope-specific CTLs were generated from HLA-A2 donors by using T2 cells as APC instead of freshly isolated PBMC or DC. It has clearly been shown that T2 cells functioned efficiently as APC in the induction of HLA-A2binding peptide-specific CTLs for its unique characteristic. For analysis of the antigen-specific CTLs induced from HLA-A2 PBMC, T2 cells would be very convenient as APCs for performing multiple experiments and for confirming reproducibility of the results in future research.

In this study, we investigated the combined immune effect of MAGE-3/HLA-A2 and MAGE-n/HLA-A2 CTL peptides. CTLs stimulated with combined epitopes can exhibit a specific lysis of HLA-A2+ tumor cells with different MAGE antigens and produce an effective anti-tumor immunity in vitro which is more effective than those stimulated with a single epitope. So this may represent a better approach in the peptide-based cancer immunotherapy. However, this study only combined MAGE-3 and MAGE-n. The combination of other MAGEderived peptides will be performed in the future.

\section{Acknowledgements}

This work was supported by grants from China National Natural Science Foundation (No. 30271464) and the Science $\&$ Technology Project of Shaanxi Province, China [No. 2007K09-05(2)].

\section{References}

1. Van den Eynde BJ and van der Bruggen P: T cell defined tumor antigens. Curr Opin Immunol 9: 684-693, 1997.

2. Thurner B, Haendle I, Roder C, et al: Vaccination with mage-3A1 peptide-pulsed mature, monocyte-derived dendritic cells expands specific cytotoxic $\mathrm{T}$ cells and induces regression of some metastases in advanced stage IV melanoma. J Exp Med 190: 1669-1678, 1999.

3. Dagher R, Long LM, Read EJ, et al: Pilot trial of tumor-specific peptide vaccination and continuous infusion interleukin-2 in patients with recurrent Ewing sarcoma and alveolar rhabdomyosarcoma: an inter-institute NIH study. Med Pediatr Oncol 38: 158-164, 2002.

4. Kawakami Y, Eliyahu S, Jennings C, et al: Recognition of multiple epitopes in the human melanoma antigen gp100 dy tumor-in-filtrating $\mathrm{T}$ lymphocytes associated with in vivo tumor regression. J Immunol 154: 3961-3968, 1995.

5. Zhang Y, Chaux P, Stroobant V, et al: A MAGE-3 peptide presented by HLA-DR1 to CD4+ T cells that were isolated from a melanoma patient vaccinated with a MAGE-3 protein. J Immunol 171: 219-225, 2003.

6. Wu W, Sui YF, Ye J, et al: The cloning of tumor-associated gene MAGE in human hepatocellular carcinoma cell line. Chin J Cell Mol Immunol 18: 270-273, 2002.

7. Dong HL, Li ZS, Ye J, et al: Identification of HLA-A2-restricted CTL epitope encoded by the MAGE-n gene of human hepatocellular carcinoma. NA: Cancer Biol Ther 3: 891-898, 2004.

8. Rosenberg SA, Yang JC, Schwartzentruber DJ, et al: Immunologic and therapeutic evaluation of a synthetic peptide vaccine for the treatment of patients with metastatic melanoma. Nat Med 4: 321-327, 1998.

9. Schaed SG, Klimek VM, Panageas KS, et al: T-cell responses against tyrosinase 368-376(370D) peptide in HLA(*)A0201(+) melanoma patients: randomized trial comparing incomplete Freund's adjuvant, granulocyte macrophage colony-stimulating factor, and QS-21 as immunological adjuvants. Clin Cancer Res 8: 967-972, 2002.

10. Salgaller ML, Marincola FM, Cormier JN and Rosenberg SA: Immunization against epitopes in the human melanoma antigen gp100 following patient immunization with synthetic peptides. Cancer Res 56: 4749-4757, 1996.

11. Slingluff CL Jr, Yamshchikov G, Neese P, et al: Phase I trial of a melanoma vaccine with gp100(280-288) peptide and tetanus helper peptide in adjuvant: immunologic and clinical outcomes. Clin Cancer Res 7: 3012-3024, 2001.

12. Scheibenbogen C, Nagorsen D, Seliger B, et al: Long-term freedom from recurrence in 2 stage IV melanoma patients following vaccination with tyrosinase peptides. Int J Cancer 99: 403-408, 2002.

13. Cormier JN, Salgaller ML, Prevette T, et al: Enhancement of cellular immunity in melanoma patients immunized with a peptide from MART-1/Melan A. Cancer J Sci Am 3: 37-44, 1997.

14. Valmori D, Dutoit V, Schnuriger V, et al: Vaccination with a Melan-A peptide selects an oligoclonal $\mathrm{T}$ cell population with increased functional avidity and tumor reactivity. J Immunol 168: 4231-4240, 2002.

15. Knutson KL, Schiffman K and Disis ML: Immunization with a HER-2/neu helper peptide vaccine generates HER-2/neu CD8 T-cell immunity in cancer patients. J Clin Invest 107: 477-484, 2001 . 
16. Khong HT and Restifo NP: Natural selection of tumor variants in the generation of 'tumor escape' phenotypes. Nat Immunol 3: 999-1005, 2002.

17. Parkhurst MR, Salgaller ML, Southwood S, et al: Improved induction of melanoma-reactive CTL with peptides from the melanoma antigen gp100 modified at HLA-A*0201-binding residues. J Immunol 6157: 2539-2548, 1996.

18. Reynolds SR, Oratz R, Shapiro RL, et al: Stimulation of CD8+ $\mathrm{T}$ cell responses to MAGE-3 and Melan A/MART-1 by immunization to a polyvalent melanoma vaccine. Int $\mathrm{J}$ Cancer 72: 972-976, 1997.

19. Ayyoub M, Mazarguil H, Monsarrat B, Van den Eynde B and Gairin JE: A structure-based approach to designing non-natural peptides that can activate antimelanoma cytotoxic T cells. J Biol Chem 274: 10227-10234, 1999.

20. Dong HL, Sui YF, Li ZS, et al: Efficient induction of cytotoxic $\mathrm{T}$ lymphocytes specific to hepatocellular carcinoma using HLA-A2-restricted MAGE-n peptide in vitro. Cancer Lett 211: 219-225, 2004.

21. Gao L, Xue SA, Hasserjian R, et al: Human cytotoxic T lymphocytes specific for Wilms' tumor antigen-1 inhibit engraftment of leukemia-initiating stem cells in non-obese diabetic-severe combined immunodeficient recipients. Transplantation 75: 1429-1436, 2003.
22. Ohta K, Yamaguchi Y, Shimizu K, Miyahara E and Toge T: Novel system for generating cytotoxic effector lymphocytes using carcinoembryonic antigen (CEA) peptide and cultured dendritic cells. Anticancer Res 22: 2597-2606, 2002.

23. Mangino G, Grazia Capri M, Barnaba V and Alberti S: Presentation of native TROP-2 tumor antigens to human cytotoxic T lymphocytes by engineered antigen-presenting cells. Int J Cancer 101: 353-359, 2002.

24. Molldrem JJ, Lee PP, Wang C, Champlin RE and Davis MM: A PR1-human leukocyte antigen-A2 tetramer can be used to isolate low-frequency cytotoxic $\mathrm{T}$ lymphocytes from healthy donors that selectively lyse chronic myelogenous leukemia. Cancer Res 59: 2675-2681, 1999.

25. Renkvist N, Castelli C, Robbins PF and Parmiani G: A listing of human tumor antigens recognized by $\mathrm{T}$ cells. Cancer Immunol Immunother 50: 3-15, 2001.

26. Riker A, Cormier J, Panelli M, Marincola F, et al: Immune selection after antigen-specific immunotherapy of melanoma. Surgery 126: 112-120, 1999.

27. Marincola FM, Jaffee EM, Hicklin DJ and Ferrone S: Escape of human solid tumors from T-cell recognition: molecular mechanisms and functional significance. Adv Immunol 74: 181-273, 2000. 